\title{
A transferência para o mercado do conhecimento produzido na pesquisa acadêmica
}

Juçara Salete Gubiani ${ }^{1}$ Aran Bey Tcholakian Morales ${ }^{2}$

Paulo Maurício Selig ${ }^{3}$

Fernando Bordin da Rocha ${ }^{4}$

\section{RESUMO}

A economia no passado centrada no capital físico, na terra, nos recursos naturais, é modificada, e a fonte da riqueza passa a ser o conhecimento. Como resultado do avanço da pesquisa em ciência e tecnologia, os padrões de produção também são modificados. Nesse contexto, as empresas que fazem a gestão do capital intelectual passam a reter e aplicar o conhecimento no desenvolvimento de novos bens e serviços, para atender a uma demanda especializada e tornam-se competitivas perante os concorrentes. No mesmo sentido, as universidades participam como agentes propulsoras da geração de conhecimento por meio da excelência na pesquisa. Nessa nova economia, centrada nos ativos do conhecimento, elas são consideradas elementos-chave e, diante da valoração do conhecimento, analisam o seu potencial de criação e transferência de conhecimento para a implementação da inovação no mercado. Em 2011, com o objetivo de testar um modelo de identificação e análise do conhecimento produzido na pesquisa das universidades, foi realizada uma pesquisa na Universidade Federal de Santa Maria, no sul do Brasil. Os resultados mostraram que a pesquisa ocorre nos laboratórios e grupos de pesquisa no contexto universitário, mas não existe um resultado inovador de relevância: a transferência do conhecimento produzido para o mercado é de baixa expressividade. A partir da aprovação da Lei de Inovação em 2004 e da implementação dos Núcleos de Inovação Tecnológica nas universidades, a tendência é uma maior aproximação da academia com o mercado na produção de conhecimento direcionado para a inovação.

Palavras-chave: Conhecimento. Capital Intelectual. Inovação. Universidades.

\section{INTRODUÇÃO}

Questões relativas à produção de conhecimento, historicamente, são relacionadas à pesquisa acadêmica das universidades. As universidades são consideradas elementos-chave no desenvolvimento econômico, na formação, na criação e transferência do conhecimento. Nos últimos anos, numa tendência mundial, a partir

\footnotetext{
${ }^{1}$ Dra. em Engenharia e Gestão do Conhecimento. Universidade Federal de Santa Maria - juçara@ufsm.br

2 Dr. em Engenharia da Produção. Universidade Federal de Santa Catarina - aran@stela.org.br

${ }^{3}$ Dr. em Engenharia da Produção. Universidade Federal de Santa Catarina - pauloselig@gmail.com

${ }^{4}$ Bel. em Engenharia Mecânica. Universidade Federal de Santa Maria - fernando.rocha@ufsm.br
} 
da valoração dos recursos do conhecimento, elas analisam o quanto mais podem fazer pela sociedade na criação de novos conhecimentos, novas tecnologias, novas competências profissionais a fim de aumentar o estoque de conhecimento na sociedade e melhorar a capacidade produtiva e o desenvolvimento regional (GOLDSTEIN; DRUCKER, 2006; GUBIANI, 2011).

A eficiência da produção de conhecimento da pesquisa acadêmica vem sendo balizada por meio das publicações; entretanto, a eficiência da aplicação desse conhecimento no mercado é hoje objeto de discussão. Do lado do mercado, as organizações estão cada vez mais preocupadas em identificar, mensurar e fazer a gestão dos seus recursos do conhecimento. Sabe-se que o conhecimento é tácito, segundo Davenport e Prusak (1998), o conhecimento é da pessoa e representa o modelo mental de cada ser humano.

As empresas fazem a gestão do conhecimento, agregam valor aos bens e serviços diferenciandose dos seus concorrentes (NONAKA; TAKEUCHI, 1997; DAVENPORT; PRUSAK, 2000). 0 conhecimento é "mais valioso e poderoso do que os recursos naturais". O que existe de comum entre as empresas bemsucedidas é o "capital intelectual". Formado pela "soma do conhecimento de todos em uma empresa, o que lhe proporciona vantagem competitiva [...] é intangível. É o conhecimento da força do trabalho" (STEWART, 1998, p. XIII).

A questão considerada neste artigo diz respeito à transferência do conhecimento produzido no contexto da pesquisa acadêmica para o mercado. Para tal, o trabalho discute a criação e a transferência do conhecimento no ambiente de pesquisa acadêmica e a sua aplicação no mercado.

0 artigo está organizado da seguinte forma: a primeira seção faz a introdução, a seção 2 discute questões relativas ao capital intelectual e à construção do conhecimento: sua criação e aplicação. A terceira detalha a metodologia usada no estudo de caso, a quarta seção discute resultados e a quinta apresenta as considerações finais desta abordagem.

\subsection{Objetivo geral}

Analisar, por meio de um estudo de caso, se as universidades transferem para o mercado o conhecimento desenvolvido no contexto da pesquisa acadêmica.

\subsection{Objetivos específicos}

a) Identificar o capital intelectual das universidades.

b) Analisar a relação existente entre os componentes do capital intelectual.

c) Concluir sobre a transferência do conhecimento produzido no contexto da pesquisa acadêmica.

\section{RECURSOS DO CONHECIMENTO}

Nas empresas, a vantagem competitiva está no uso estratégico dos recursos do conhecimento (PETRASH, 1996; KAPLAN; NORTON, 1997; 2004; ROOS; ROOS, 1997; EDVINSSON; MALONE, 1998; SVEIBY, 1998; STEWART, 1998; BONTIS, 1999; HII; NEELY, 2000; KLEIN, 2002; GONZÁLEZ; SALLERO, 2010).

Sabe-se que, nas universidades, o objetivo não é o lucro e, no Brasil, a competitividade é medida por meio de avaliações externas: publicação científica e qualificação dos egressos. Nos países desenvolvidos, a realidade é outra, e as empresas são parceiras na produção e transferência do conhecimento produzido na pesquisa. Segundo Drucker (2002), as empresas investem parte de seus recursos econômicos na produção e disseminação de conhecimento, fato comprovado pela Organização para a Cooperação e Desenvolvimento Econômico (OCDE).

Nesse contexto, nos Estados Unidos e em alguns países da Europa, as universidades discutem sobre o desenvolvimento regional e buscam formas de atender às crescentes demandas regionais por meio da aplicação de novas tecnologias na implementação da inovação (TERRA; ETZKOWITZ, 1998; ROLIM; SERRA, 2009; GOLDSTEIN, 2005; GOLDSTEIN; RENAULT, 2005; GOLDSTEIN; DRUCKER, 2006; GOLDSTEIN; DRUCKER, 2007; ARBO; BENNEWORTH, 2007; GODDARD; PUUKKA, 2008; DUCH; GARCÍA; PARELLADA, 2008; GUBIANI, 2011).

De acordo com Etzkowitz (2009, p. 37), “a capitalização do conhecimento está no cerne de uma nova missão para a universidade, a de conectar-se aos usuários do conhecimento de forma mais próxima e estabelecer-se como um ator econômico por mérito próprio". O papel da universidade é identificar problemas e explorar os potenciais da pesquisa - uma incubadora natural - com condições de dar suporte a professores e alunos para eles empreenderem. 


\subsection{0 capital intelectual}

O capital intelectual tem sido debatido nas ultimas décadas e continua em evidência na atualidade. A crescente importância está associada ao advento da economia do conhecimento, juntamente com o reconhecimento pela comunidade científica e empresarial, do impacto político do conhecimento no desempenho de indivíduos, empresas e países. A importância do conhecimento, como motor da competitividade, estimula o desenvolvimento de pesquisas sobre o capital intelectual (RODRIGUES; DORREGO; JARDÓM-FERNÁNDEZ; 2009).

O conhecimento, as experiências, a especialização e os diversos ativos intangíveis disponíveis formam o capital intelectual das empresas (KLEIN, 2002). Não somente a capacidade intelectual humana como também os produtos e marcas registradas, ativos contabilizados a custo histórico e que hoje possuem valor (EDVINSSON; MALONE, 1998). Todos os ativos - tangíveis e intangíveis - originam-se no pessoal da organização (SVEIBY, 1998).

É a força de trabalho que pode ser utilizada para gerar riqueza: o treinamento e a intuição de uma equipe, o know-how de trabalhadores que melhoram a eficácia da empresa, a tecnologia que favorece a comunicação, a cooperação, o aprendizado compartilhado interno e externo à empresa (STEWART, 1998).

Capital Intelectual combina o conhecimento das pessoas em diferentes áreas do conhecimento, na multidisciplinaridade de conteúdos, nas relações e interações sociais das pessoas em um ambiente propício para a criação do conhecimento no contexto estrutural existente (GUBIANI, 2011).

A OCDE refere-se à gestão do conhecimento como a gestão do capital intelectual, das competências organizacionais e do capital humano disponível numa organização (OECD, 1999). São muitas as definições de capital intelectual encontradas na literatura e todas, de alguma forma, abordam os três capitais: capital humano (individual e coletivo); capital estrutural/organizativo (infraestrutura física e tecnológica da organização) e capital relacional (clientes, fornecedores e a rede interna e externa) (PETRASH, 1996; KAPLAN; NORTON, 1997; 2004; STEWART, 1998; EDVINSSON; MALONE, 1998; STEWART, 1998; SVEIBY, 1998; BONTIS, 1999; BONTIS; KEOW; RICHARDSON, 2000; ROOS; ROOS, 1997; GONZÁLEZ; SALLERO, 2010).

Conforme mostra a Figura 1, a parte "que pensa", o capital humano são os aspectos relacionados à pessoa, as competências e habilidades acumuladas, as capacidades individuais e dos grupos, as experiências e os conhecimentos pessoais na organização, a educação, a agilidade intelectual, a capacidade criativa de inovação, os valores e a motivação/atitudes. A "que não pensa", capital estrutural são os aspectos internos da organização (GONZÁLEZ; SALLERO, 2010).

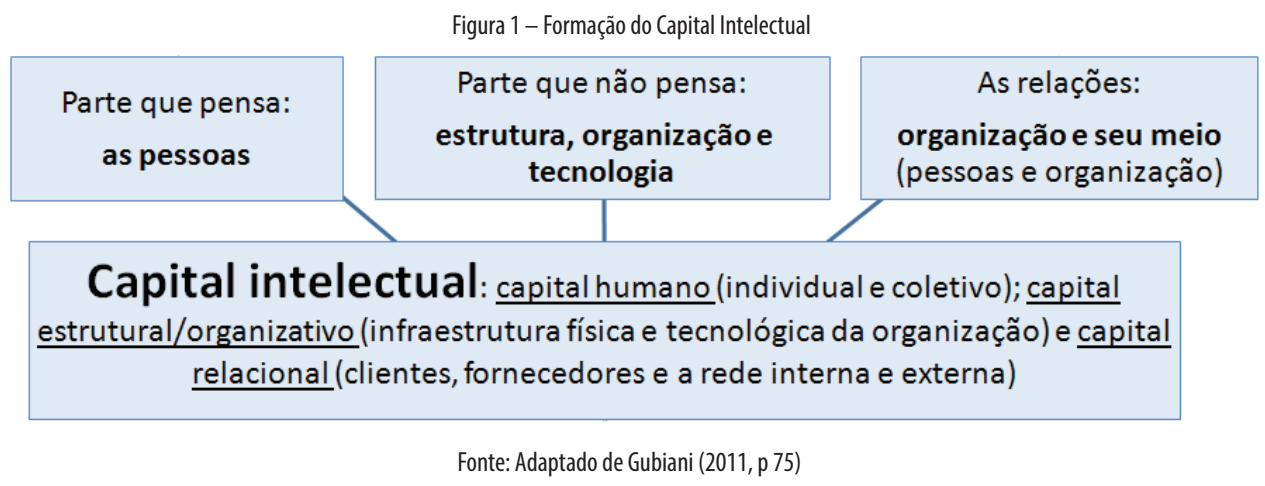

Saint-Onge (1996) esclarece que o capital humano constrói o capital estrutural; quanto melhor for o capital estrutural, melhor são as perspectivas do capital humano. 0 capital estrutural são todos os ativos intangíveis capturados pela estrutura organizacional e responsáveis pelo desenvolvimento das atividades da empresa (BONTIS, 1999; EDVINSSON; MALONE, 1998; STEWART, 1998; YOUNDT; SUBRAMANIAM; SNELL, 2004).

Por último, o capital relacional é uma medida que diz respeito ao conjunto de ativos, normalmente de caráter intangível, que são resultado da interação da empresa com o seu meio. Alguns autores explicam que esse capital é humano e a diferença está no fato de ele ser próprio da empresa (KAPLAN; NORTON, 2004; 1997; BONTIS, 1998; 1999; EDVINSSON; SULLIVAN, 1996; EDVINSSON; MALONE, 1998; STEWART, 1998; SVEIBY, 1998; SVEIBY; SIMONS, 2002; YOUNDT; SUBRAMANIAM; SNELL, 2004, 2004; LLAUGER, 2001; HII; NEELY, 2000; DAVILA; EPSTEIN; SHELTON, 2007). 


\subsection{A produção do conhecimento nas universidades}

Os países desenvolvidos investem parte de seus recursos econômicos na produção e disseminação de conhecimento, fato comprovado pela OCDE. Por outro lado, segundo publicação do Instituto Brasileiro de Geografia e Estatística (IBGE) e levantamento realizado pela Pesquisa Industrial de Inovação Tecnológica (PINTEC), países em desenvolvimento, como o Brasil, por exemplo, os investimentos em conhecimento são modestos, resultando num processo limitado de inovação. 0 baixo nível de inovação não gera recursos econômicos e sociais suficientes para o desenvolvimento do ciclo "virtuoso" na pesquisa-inovação (SILVA, 2005; BERGERMAN, 2005).

A academia produz e publica pesquisas em grande quantidade, o Brasil aparece entre os vinte países que mais publicam, sendo responsável por $1,92 \%$ e, no entanto, responde por menos de $0,1 \%$ das patentes concedidas nos mercados competitivos em 2005. A conversão do conhecimento de pesquisa em produtos e serviços praticamente não ocorre (BERGERMAN, 2005). Em 2010, segundo o Ministério da Ciência, Tecnologia e Inovação (MCTI), em nível mundial, o Brasil foi responsável por 2,1\% de todas as publicações e $0,2 \%$ do registro de patentes.

Em países desenvolvidos, empresas investem alto na contratação de cientistas e engenheiros, financiando seus próprios laboratórios corporativos de P\&D e protegendo suas inovações por meio do registro de propriedade intelectual (BERGERMAN, 2005). 0 setor privado realiza a inovação e oferece ao mercado novos produtos, serviços ou versões melhoradas destes. No Brasil, mais de $70 \%$ dos pesquisadores estão dentro das universidades em tempo integral e não adquirem qualquer experiência profissional (ROCHA NETO, 2005).

Nos últimos anos, os institutos privados de pesquisa aparecem como produtores de conhecimento e mantêm um corpo técnico multidisciplinar de alto nível atendendo integral ou parcialmente a todos os requisitos exigidos de um agente de inovação. Em 2009, o Conselho Nacional de Desenvolvimento Científico e Tecnológico (CNPq) estabeleceu a criação de novos centros de excelência em pesquisas básica e aplicada, distribuídos por todo o território nacional e atualmente são 123 Institutos Nacionais de Ciência e Tecnologia (INCT).

Com essa ação, o MCTI propôs a criação de centros de excelência para impulsionar a pesquisa científica básica e fundamental, gerando e estimulando o desenvolvimento de pesquisa científica e tecnológica de ponta para promover a inovação e o espírito empreendedor em conjunto com empresas inovadoras nas áreas de tecnologia.

Nesse sentido, em uma tendência mundial, as universidades gradativamente modificam sua atuação na sociedade e na economia, ultrapassando o espaço acadêmico antes direcionado para a realização de pesquisa puramente acadêmica (TERRA; ETZKOWITZ, 1998; ROLIM; SERRA, 2009; GOLDSTEIN, 2005; GOLDSTEIN; RENAULT, 2005; GOLDSTEIN; DRUCKER, 2006; GOLDSTEIN; DRUCKER, 2007; ARBO; BENNEWORTH, 2007; GODDARD; PUUKKA, 2008; DUCH; GARCÍA; PARELLADA, 2008).

São criados escritórios de transferência de tecnologia com a finalidade de capturar o conhecimento e a tecnologia dos grupos de pesquisa para a alocação no mercado produtivo. No último estágio da evolução da transferência, o conhecimento e a tecnologia são incorporados em uma empresa e retirados da universidade por um empreendedor.

Relativamente à proteção, nos Estados Unidos (EUA), a lei Bayh-Dole, aprovada em 1980, criou mecanismos oficiais para regulamentar o conhecimento desenvolvido nas universidades públicas. No Brasil, a Lei de Inovação aprovada em dezembro de 2004 (Lei Federal no 10.973, de 2 de dezembro de 2004), do Ministério da Ciência e Tecnologia, estabelece mudanças nas universidades no tocante à relação universidades e institutos de pesquisa, empresas e o governo. A Lei "Dispõe sobre incentivos à inovação e à pesquisa científica e tecnológica no ambiente produtivo e dá outras providências". A lei regulamenta as parcerias estratégicas entre as universidades, institutos tecnológicos e empresas.

Estudos realizados em universidades americanas e europeias demonstraram que muitas universidades desenvolvem iniciativas políticas destinadas a aumentar sua participação no desenvolvimento regional. 0 empreendedorismo acadêmico, as redes de conhecimento e os clusters de desenvolvimento aproximam cada vez mais a universidade na sua região. Elas buscam políticas baseadas em "suposições" sobre os papéis que elas podem ou devem exercer na economia e atender, de forma efetiva e ampla, às demandas regionais crescentes (HUGGINS; JOHNSTON; STEFFENSON, 2008; DRUCKER; GOLDSTEIN 2007).

Entretanto, a universidade brasileira, aparentemente, ainda está pouco sintonizada com as necessidades da sociedade e longe de criar um sistema de inovação que consiga efetivamente conectar e articular os diferentes atores e setores que deveriam integrar o sistema: governo, setor público e empresarial, comunidade científica e universidades (SCHWWARZMAN, 2008). 


\section{PROCEDIMENTOS METODOLÓGICOS}

Este estudo mostra parte dos resultados de uma pesquisa de cunho exploratório, cujo objetivo foi a validação de um modelo desenvolvido em uma tese de doutorado defendida em 2011 na Universidade Federal de Santa Catarina no Programa de Pós-Graduação em Engenharia do Conhecimento. A pesquisa foi realizada na Universidade Federal de Santa Maria, situada no estado do Rio Grande do Sul, Brasil (GUBIANI, 2011).

O modelo proposto na tese analisa o potencial de criação de conhecimento das universidades e seu resultado por meio da implementação da inovação. Os dados primários do estudo foram coletados via questionário disponibilizado no portal dos professores, para todos os professores efetivos admitidos até dezembro de 2008 na Universidade e que, naquele momento, estivessem atuando na pesquisa. Para a tabulação e análise dos dados da pesquisa, foram utilizados recursos computacionais e ferramentas estatísticas. Para realizar a análise multivariada, utilizou-se o software SPSS versão 18.

\subsection{Materiais}

A tese de Gubiani (2011) definiu um modelo para a análise do conhecimento produzido no contexto das universidades. 0 modelo tem como arcabouço conceitual os trabalhos desenvolvidos por pesquisadores vinculados a universidades americanas, europeias, canadenses e pesquisadores da OCDE (SVEIBY, 1998; EDVINSSON; MALONE, 1998; STEWART, 1998; BONTIS, 1998; 1999, KAPLAN; NORTON, 1997; 2004; ROOS; ROOS, 1997; HII; NEELY, 2000; LLAUGER, 2001; GOLDSTEIN; RENAULT, 2005; ARBO; BENWORTH, 2007; GODDARD; PUUKKA, 2008; RODRIGUES; DORREGO; JARDÓM-FERNÁNDEZ, 2009; LENDEL, 2010).

0 modelo orienta para uma relação positiva e significativa entre os componentes do capital intelectual relacionados na tese: capital humano, capital estrutural e capital relacional. A tese defende que o capital intelectual é o resultado do intercâmbio destes três elementos: capital humano, capital estrutural e capital de clientes.

0 artigo aborda a transferência do conhecimento produzido no contexto da pesquisa na universidade para a sociedade. Nas universidades, diferentemente das empresas, a ideia comercial da inovação não está presente, sendo assim, o trabalho não relaciona o conceito de capacidade de inovação na dimensão da universidade e define, para as universidades, o conceito de potencial de inovação pela transferência do conhecimento produzido pelo capital intelectual da universidade.

A Figura 2 mostra o modelo teórico de análise usado na tese e base deste artigo.

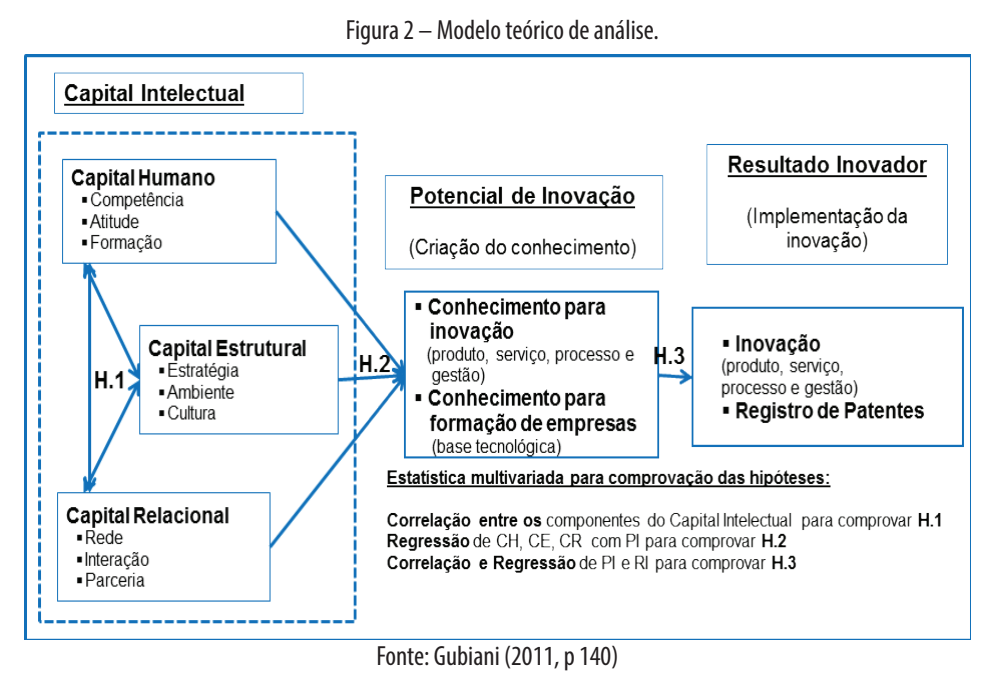

\subsection{Métodos}

O método reporta-se aos procedimentos metodológicos usados na tese defendida por Gubiani (2011). Inicia com análise fatorial exploratória com o intuito de reduzir as variáveis observadas; na sequência, identifica as cargas fatoriais, executa a análise da confiabilidade dos constructos para obter a proposta teórica (PESTANA; GAGEIRO, 2003). 
O modelo pressupõe o uso de correlação entre as variáveis; contudo, estatisticamente, uma correlação, tanto positiva quanto negativa entre duas variáveis apenas mostra que as duas crescem no mesmo sentido. 0 retorno indica o grau de relacionamento entre variáveis, mas não a influência de uma sobre a outra, ou seja, não informa sobre o poder preditivo das variáveis envolvidas na relação (HAIR et al, 2009).

Para realizar a predição de uma ou mais variáveis sobre outra, a técnica recomendada é a regressão ou equações estruturais. Para demonstrar a existência ou não da transferência do conhecimento pesquisado para a sociedade, foi aplicado regressão múltipla, a qual fornece as predições de um resultado, partindo de uma ou mais variáveis previsoras. A associação entre as variáveis independentes com a variável dependente pode ser verificada pelo $R^{2}$ ou $R^{2}$ ajustado na regressão (HAIR et al, 2009).

\section{DISCUSSÃO DOS RESULTADOS}

O objetivo deste estudo é discutir questões acerca do conhecimento desenvolvido no ambiente de pesquisa das universidades e sua efetiva transferência para o mercado. Dessa forma, para a verificação da existência de transferência do conhecimento para a sociedade e seus prováveis impactos, a análise foi efetuada tomando-se por base o resultado inovador com base nas respostas dos pesquisadores entrevistados.

Nesse aspecto, duas análises estatísticas foram realizadas: na primeira, aplicou-se a técnica estatística de correlação com o objetivo de identificar a relação entre os constructos do capital humano, capital estrutural e capital relacional identificados no processo anterior (análise fatorial exploratória) e a significância destes; na segunda, aplicou-se regressão múltipla com o intuito de concluir sobre o resultado inovador: verificar a existência de transferência de conhecimento para a implementação da inovação no mercado.

\subsection{Relação entre os capitais}

A relação entre os três capitais (capital humano, capital estrutural e capital de relacional) forma o capital intelectual e a base de criação de valor nas organizações. 0 capital humano é responsável por construir e alimentar o capital estrutural e este, por sua vez, incorpora, capacita e apoia o capital humano - o agente capaz de assimilar, processar e disseminar conhecimento. 0 capital relacional é baseado no relacionamento com pessoas de fora da organização; assim, ele é mais individual do que organizacional. Não é possível considerar o capital relacional sem prever a influência do capital humano sobre este.

O modelo teórico de análise proposto na tese define as hipóteses sobre as relações, e o coeficiente de correlação de Pearson fornece o grau de significância para cada relação. A proposta da correlação é identificar se há relação entre os constructos elencados no modelo. Bons coeficientes fornecem condições para interpretar e concluir a existência sobre o potencial para criação do conhecimento, mas não sobre a eficiência dessa produção. A correlação apontou os constructos: Atitude, Competência, Formação, Estratégia, Ambiente, Cultura, Interação, Parceria, Rede.

0 coeficiente mede a força da relação, e os valores mostram uma relação de fraca a moderada. Mesmo assim, o resultado encontrado reforça o conceito de que o capital humano influencia positivamente no capital estrutural inovando e renovando a base do conhecimento da empresa (DAVENPORT; PRUSAK; WILSON, 2003).

A parte que "pensa", o capital humano, relaciona-se com o capital estrutural e é influenciada pelo segundo. 0 capital intelectual, como medida do conhecimento, precisa da intervenção do capital humano que influencia a parte que não pensa, com atitudes, competência, conhecimento e agilidade intelectual.

A Figura 3 mostra o nível do coeficiente de correlação de Pearson com significância $(p<0,05)$. 


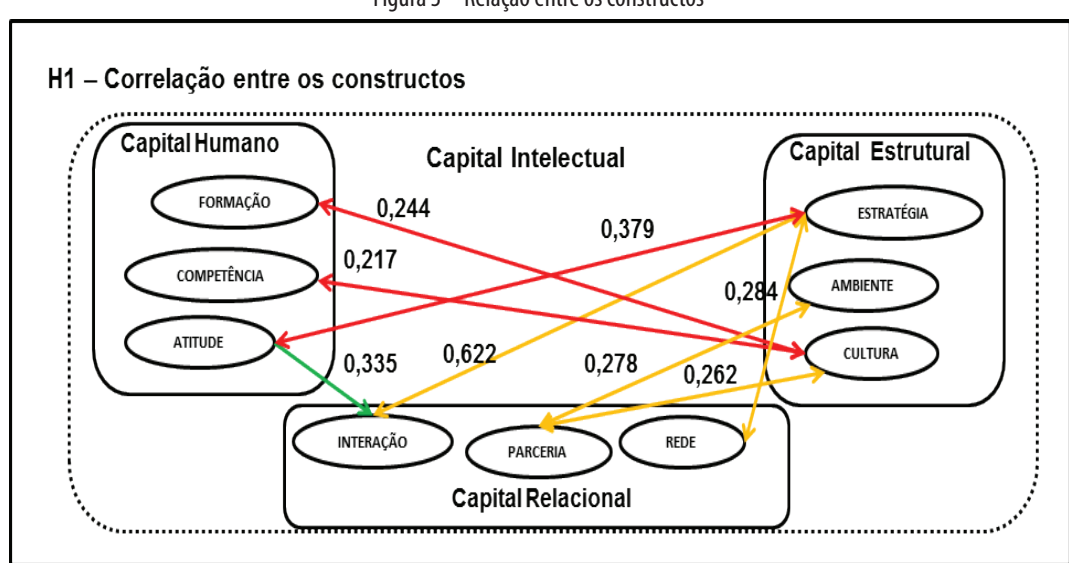

Fonte: Gubiani (2011, p 142)

O capital humano ajuda a construir o capital estrutural e, ao influenciar o capital estrutural, o capital humano também é melhorado. Nesse raciocínio, as competências individuais somente servem à empresa se puderem ser transformadas em recursos disponíveis para a criação do conhecimento. Estudos comprovam a influência do capital humano no capital estrutural: no Canadá (BONTIS, 1998), na Malásia (BONTIS; KEOW; RICHARDSON; 2000), em Portugal (CURADO, 2006), na Espanha (RODRIGUES; DORREGO; JARDÓMFERNÁNDEZ, 2009).

A literatura afirma que o capital relacional é mais humano do que da empresa. No caso especifico do ambiente acadêmico, a atitude de criar um conhecimento novo é de cunho pessoal e pouco relacionado à estrutura da universidade. 0 apoio necessário para a prática da inovação não é institucional, diferentemente das empresas que visam ao lucro e à competitividade. Os pesquisadores americanos (LYNCH; AYDIN, 2004; GOLDSTEIN; DRUCKER, 2006) também concluíram que a conexão com as demandas da sociedade exige gestão acadêmica dos recursos intangíveis para, em conjunto, planejar a produção e contribuição para o desenvolvimento de suas regiões.

\subsection{A transferência do conhecimento}

Na identificação do diagnóstico da influência do potencial de inovação no resultado inovador, duas análises estatísticas foram realizadas: a primeira foi uma correlação com o escopo de identificar a relação e a significância dos constructos a partir da base necessária para a criação do conhecimento, ou seja: anteriormente, concluiu-se sobre a existência de potencial para a criação medido anteriormente para relação entre os capitais; na segunda, aplicou-se a técnica de regressão múltipla, analisando o resultado inovador diante do potencial de criação do conhecimento para a inovação.

A regressão explica o nível de transferência de conhecimento criado para aplicação na inovação. Dois testes foram realizados, o primeiro cujo fim era analisar a criação para a transferência por meio da criação de empresas de base tecnológica e o segundo por meio do registro de patentes.

No primeiro teste, o coeficiente de determinação $\mathrm{R}^{2}$ explicou 14,6\% da variância do resultado inovador (variável dependente que identifica a transferência do conhecimento). Isso significa que as variáveis independentes - criação do conhecimento para a inovação e para a criação de empresa (previsores do modelo) - explicam somente $14,6 \%$ da variância do resultado inovador (transferência do conhecimento).

No segundo teste, o coeficiente de determinação $\mathrm{R}^{2}$ retornou 13,3\% da variância do resultado inovador na variável registro de patentes (variável dependente patente). Em tese, esse resultado explica somente $13,3 \%$ da variância do resultado medido pelo registro de patentes definida no estudo,

Os números apontam que a pesquisa ocorre, mas não existe resultado inovador de relevância. A dimensão que avalia o resultado inovador demonstrou que, aproximadamente, $40 \%$ dos respondentes desconhecem a existência da implementação da inovação no mercado, da formação de empresas e do registro de patentes.

Diante do resultado das relações e coeficientes identificados nas análises, tornou-se possível concluir estatisticamente que os resultados inovadores correspondem a diferentes potenciais de inovação. 


\title{
5 CONCLUSÃO
}

As universidades são estruturas organizacionais complexas e, no Brasil, geralmente, atuam na tríade ensino, pesquisa e extensão em todas as áreas do conhecimento. 0 pressuposto norteador do estudo foi de que, ao atuarem na pesquisa, na criação do conhecimento, elas participam como agentes de inovação nos sistemas regionais de inovação.

Essa afirmação é confirmada ao caracterizar o ambiente produtivo para a verificação de consistência do modelo. Os dados da pesquisa documental possibilitaram concluir que o ambiente da pesquisa, ao logo do tempo, vem sendo melhorado e oferece condições para formar um estoque de conhecimento e base para a criação do conhecimento.

A literatura é unânime ao afirmar que o papel das universidades, no modelo econômico vigente, vai muito além da formação acadêmica e da pesquisa básica. 0 resultado encontrado, no estudo de caso, mostra que a Universidade, ainda que moderadamente, busca mecanismos para a criação de conhecimento aplicado às demandas da sociedade.

O estudo considera que a inovação ocorre no mercado pela transferência do conhecimento criado na pesquisa aos agentes econômicos. O capital intelectual é o responsável por formar o estoque de conhecimento, entretanto, o estudo de caso forneceu índices baixos para o resultado inovador. Os números obtidos por meio de uma frequência mostraram que a pesquisa ocorre, no entanto, a análise de regressão comprova uma baixa ou inexistência da transferência do conhecimento para o mercado (inovação). Esse resultado contraria dados encontrados na literatura internacional, a qual relata que, a partir do potencial de criação do conhecimento, existe transferência para a sociedade por meio da implementação da inovação no mercado.

Os resultados encontrados orientam para uma baixa conexão da pesquisa com o mercado. 0 instrumento aplicado na UFSM mostrou que a cultura da Universidade ainda não é para a criação do conhecimento para a inovação no mercado. Essa situação tende a se modificar diante da aprovação da Lei de Inovação em 2004 e da implementação dos Núcleos de Inovação Tecnológica nas universidades.

Como sugestão para trabalhos futuros, aponta-se a análise na dimensão das universidades, as dificuldades encontradas pelos docentes na execução das pesquisas; análise do conhecimento coletivo e do individual na criação do conhecimento para a inovação da visão do aluno de pós-graduação; análise da maturação das empresas de base tecnológicas formadas pelo conhecimento criado na pesquisa acadêmica e; partindo da proposta aplicada neste trabalho, uso de outras técnicas de análise estatística para inferir resultados como, por exemplo, modelos de equações estruturais.

\section{THE TRANSFER TO THE KNOWLEDGE MARKET PRODUCED IN ACADEMIC RESEARCH}

\begin{abstract}
The economy in the past focused on physical capital, land, natural resources, is modified and the source of wealth becomes the knowledge. As a result of research advances in science and technology, production patterns are also modified. In this context, the companies that make the management of intellectual capital, retain and apply the knowledge to develop new goods and services, to meet a demand specialized and become competitive against competitors. Similarly, the participating universities as agents driving the generation of knowledge through excellence in research. In this new economy focused on knowledge assets, they are considered key elements and, facing with the knowledge appraisement, the potential for the creation and transfer of knowledge to the implementation of innovation in the market has been analyzed. In 2011, in order to test a model for identifying and analyzing the knowledge produced in university research, a survey was carried out at the Federal University of Santa Maria, in the South of Brazil. The results showed takes place in the laboratories and research groups in academic contexts, but innovative result has no relevance: the transfer of the knowledge produced for the market is low expressiveness. Since the approval of the Innovation Law in 2004 and the implementation of the Technological Innovation in universities, the tendency is a further approximation of academy with the market directed towards the production of knowledge for innovation.
\end{abstract}

Keywords: Knowledge. Intellectual Capital. Innovation. Universities 


\section{REFERÊNCIAS}

ARBO, P.; BENNWORTH, P. Understanding the Regional Contribution of Higher Education Institutions: A Literature Review. OECD Education Working Paper, Danvers, MA, n. 9, 2007.

BERGERMAN, M. Inovação como instrumento de geração de riqueza no Brasil: o exemplo dos institutos privados de inovação tecnológica. Parcerias Estratégicas, Brasília, n. 20, p. 1419-1428, 2005.

BONTIS, N. Intellectual capital: an exploratory study that develops measures and models. Management Decision, University of Western Ontario, Ontario, Canada, v. 36, n. 2, p. 63-76, 1998.

.Managing Organizational Knowledge by Diagnosing Intellectual Capital: Framing and advancing the state of the field. International Journal of Technology Management, Canada, v. 18, n. 5, p. 433-462, 1999.

BONTIS, N.; KEOW, W. C. C.; RICHARDSON, S. Intellectual Capital and business performance in Malaysian industries. Journal of Intellectual Capital, Malaysian, v. 1, n. 1, p. 85-100, 2000.

CURADO, C. M. M. 0 efeito mediador das estratégias de gestão do conhecimento entre componentes do Capital Intelectual: Um estudo realizado na indústria bancária portuguesa. 2006.Tese (Doutorado) Universidade Técnica de Lisboa, Lisboa.

DAVENPORT, T. H.; PRUSAK, L.; WILSON, H. J. Who's bringing you hot ideas and are you responding? Harvard Business School Press, Massachusetts, USA, v. 81, n. 2, p. 58-64, 2003.

DAVENPORT, T.; PRUSAK, L. Conhecimento empresarial. Rio de Janeiro: Campus, 1998.

.Working Knowledge: How Organizations Manage What They Know. Boston: Harvard Business Scholl Press, 2000.

DAVILA, T.; EPSTEIN, M. J.; SHELTON, R. As Regras da Inovação. Porto Alegre: Bookman, 2007.

DRUCKER, J; GOLDSTEIN, H. Assessing the Regional Economic Development Impacts of Universities: A Review of Current Approaches. International Regional Science Review, Sage Publications, Thousand Oaks, v. 30, n. 20, 2007.

DRUCKER, P. Sociedade pós-capitalista. São Paulo: Pioneira, 2002.

DUCH, N.; GARCIA, J.; PARELLADA, M. The Economic Impact of the Spanish Public University System: An Analysis for the Period 1998 - 2004. Barcelona: IEB Institute d’Economia de Barcelona, 2008.

EDVINSSON, L.; MALONE, M. S. Capital Intelectual. São Paulo: Makron Books, 1998.

EDVINSSON, L.; SULLIVAN, P. Developing a model for managing Intellectual Capital. European Management Journal, California, v. 14, n. 4, p. 356-364,1996.

ETZKOWITZ, H. Hélice Tríplice: Universidade-Indústria-Governo - Inovação em Movimento. Porto Alegre: EDIPUCRS, 2009.

GODDARD, J; PUUKKA, J. The Engagement of Higher Education Institutions in Regional Development: An Overview of the Opportunities and Challenges, Higher Education Management and Policy. The journal of OECD's Programme on Institutional Management in Higher Education, Paris, v. 20, n. 2, 2008. 
GOLDSTEIN, H. What We Know and Don't Know About the Regional Economic Impacts of Universities. Workshop on Universities and Regional Development: success factors and dangers of failure, Pecs, Hungary, 2005. Annals... Pecs: University of Pecs, 2005.

GOLDSTEIN, Harvey; DRUCKER, Joshua. The economic development impacts of universities on regions: do size and distance matter? Economic development quarterly, [S.l.], v. 20, n. 1, p. 22-43, 2006.

. Assessing the regional economic development impacts of universities: a review of current approaches. International regional science review, [S.l.], v. 30, n. 1, p. 20-46, 2007.

GOLDSTEIN, H.; RENAULT, C. S. Estimating Universities Contributions to Regional Economic Development: The Case of the U.S., Spillovers and Innovations. New York: Springer, 2005.

GONZÁLEZ, M. M. C; SALLERO, F. J. S. Gestão do conhecimento na gestão estratégica dos recursos humanos no setor da aqüicultura da Espanha. Revista Brasileira de Gestão e Desenvolvimento Regional, Taubaté, v. 6, n. 1, p. 137-164, 2010.

GUBIANI, J. S. Modelo para Diagnosticar a Influência do Capital Intelectual no Potencial de Inovação nas Universidades. 2011. Tese (Doutorado) - Universidade Federal de Santa Catarina, Florianópolis.

HAIR, J. F. et al. Análise Multivariada de Dados. 6. ed. Porto Alegre: Bookman, 2009.

HII, J.; NEELY, N. Innovative capacity of firms: on why some firms are more innovative than others. [2000]. Disponível em: <https://dspace.lib.cranfield.ac.uk/bitstream/1826/3788/3/Innovative_capacity_ of_firms-2000.pdf>. Acesso em: 18 out. 2013.

HUGGINS, R.; JOHNSTON, A.; STEFFENSON, R. Universities, knowledge networks and regional policy, Cambridge Journal of Regions. Economy and Society, Oxford, v. 1, n. 2, 321-340, 2008.

KAPLAN, R. S.; NORTON, D. P. A Estratégia em Ação: Balanced Scorecard. Rio de Janeiro: Campus, 1997.

A Strategy Maps: Converting Intangible Assets into Tangible Outcomes. Boston: Harvard Business Publishing Corporation, 2004.

KLEIN, D. A Gestão Estratégica do Capital Intelectual. Rio de Janeiro: Qualitymark, 2002.

LENDEL, I. The Impact of Research Universities on Regional Economies: The Concept of University Products. Economic Development Quarterly, Cleveland, v. 24, n. 3, p. 210-230, Aug. 2010.

LLAUGER, M. B. Hacia la economía del conocimiento. Madrid: ESIC Editorial PricewaterhouseCooper, 2001. Disponível em: <http://books.google.com.br. Acesso em: 10 jul. 2013.

LYNCH ,T.; AYDIN, N. Literature Review of the Economic and Social Impact of Higher Education Research Funding. Tallahassee: Florida State University, 2004.

NONAKA, I.; TAKEUCHI, H. Criação de Conhecimento na Empresa: como as empresas japonesas geram a dinâmica da inovação. Rio de Janeiro: Campus, 1997.

ORGANISATION FOR ECONOMIC CO-OPERATION AND DEVELOPMENT (OECD). Managing National Innovation Systems. Paris: OECD, 1999. 
PESTANA, M. H.; GAGEIRO, J. N. Análise de dados para ciências sociais: a complementaridade do SPSS. 3. ed. Lisboa: Sílabo, 2003.

PETRASH, G. Dow's journey to a knowledge value management culture. European Management Journal Elsevier, Great Britain, v. 14, n. 4, p. 365-373, 1996.

ROCHA NETO, I. Regionalização de C\&T e geração de riqueza. In: 3a Conferência Nacional de Ciência, Tecnologia e Inovação: síntese das conclusões e recomendações. Brasília: Ministério da Ciência e Tecnologia, 2006. p. 63-79.

RODRIGUES, H. M. S. S.; DORREGO, P. F. F.; JARDÓM-FERNÁNDEZ, C. M. F., En la Capacidad de Innovación de las Empresas del Sector de Automoción de la Eurorregión Galicia Norte de Portugal, 2009. (Tese) Doutorado - Universidade de Vigo, Vigo.

ROLIM, C. F. C.; SERRA, M. A. Universidade e desenvolvimento regional: o apoio das Instituições de Ensino Superior ao Desenvolvimento Regional. Curitiba: Juruá, 2009.

ROOS, G.; R00S, J. Measuring your company's Intellectual performance. Long Range Planning, Great Britain, v. 30, n. 3, p. 413-426, 1997.

SAINT-ONGE, H. Tacit knowledge: The key to the strategic alignment of Intellectual Capital. Strategy and Leadership, United Kingdom, v. 24, n. 2, p. 10-14, 1996.

SCHWWARZMAN, S. Pesquisa universitária e inovação no Brasil. Brasília: Centro de Gestão e Estudos Estratégicos, Ciência, Tecnologia e Inovação, 2008.

SILVA, E. M. P. Modelo de Inserção de C,T\&I para o desenvolvimento nacional. Revista Parcerias Estratégicas, Brasília, n. 20, p. 1339-1347, jun. 2005.

STEWART, T. A. Capital intelectual: a nova vantagem competitiva das empresas. Rio de Janeiro: Campus, 1998.

SVEIBY, K. E. A Nova Riqueza das Organizações: gerenciando e avaliando patrimônios do conhecimento. Rio de Janeiro: Campus, 1998.

SVEIBY, K.-E.; SIMONS, R. Collaborative climate and effectiveness of knowledge work: an empirical study. Journal of Knowledge Management, United Kingdom, v. 6, n. 5, p. 420-433, 2002.

TERRA, B.; ETZKOWITZ, H. A universidade empreendedora e a sociedade da nova era.[1998]. Disponível em: <http://www.competenet.org.br/evento/branca.pdf>. Acesso em: 10 jul. 2013.

YOUNDT, M. A.; SUBRAMANIAM, M.; SNELL, S. A. Intellectual Capital profiles: an examination of investments and returns. Journal of Management Studies, Malden, v. 41, n. 2, p. 335-361, 2004. 